\title{
Structural Properties of Laterally Overgrown GaN
}

\author{
R. Zhang, Y, Shi, Y.G. Zhou, B. Shen, Y.D. Zheng \\ Laboratory of Solid State Microstructures and Department of Physics, Nanjing \\ University, Nanjing 210093, CHINA \\ T.S. Kuan \\ Department of Physics, State University of New York at Albany, Albany, NY 12222 \\ S.L. Gu, L. Zhang, D.M. Hansen and T.F. Kuech \\ Department of Chemical Engineering, University of Wisconsin, Madison, WI 53706
}

\begin{abstract}
Structural properties of epitaxially laterally overgrown (ELO) GaN on patterned $\mathrm{GaN}$ 'substrates' by hydride vapor phase epitaxy (HVPE) have been investigated. The epitaxially lateral overgrowth of $\mathrm{GaN}$ on $\mathrm{SiO}_{2}$ areas is realized and a planar $\mathrm{ELO} \mathrm{GaN}$ film is obtained. Scanning electron microscope, transmission electron microscope (TEM) and atomic force microscope (AFM) are used to study the structure and surface morphology of the ELO GaN materials. AFM images indicate that no observable step termination is detected over a $4 \mu \mathrm{m}^{2}$ area in the ELO region. TEM observations indicate that the dislocation density is very low in the ELO region. No void at the coalescence interface is observed. Lattice bending as high as $3.3^{\circ}$ is observed and attributed to pileup of threading dislocations coming from the underlying $\mathrm{GaN}$ "seeding layer" and tilting horizontally and quenching at the coalescence interface.
\end{abstract}

\section{INTRODUCTION}

$\mathrm{GaN}$ and related compound materials have been intensively studied due to their important practical applications and promising for potential applications [1-3]. The existence of high density threading dislocations in $\mathrm{GaN}$ films is one of major factors to impede further development of GaN optoelectronic and electronic devices [4]. So far the most successful and practical technique for reducing the threading dislocation (TD) density of GaN epilayers is the epitaxial lateral overgrowth (ELO). The TD density of the $\mathrm{GaN}$ ELO region could be 4 or more orders lower than the conventionally grown $\mathrm{GaN}$ films [5]. Both metalorganic vapor phase epitaxy (MOVPE)[6-9] and hydride vapor phase epitaxy (HVPE)[10-11] have been used to produce GaN ELO materials with high crystal quality. HVPE is a very useful technique to grow $\mathrm{GaN}$ films via both large-area epitaxy and ELO. In HVPE ELO GaN materials, no observable void is found in the coalescence interface and a variety of growth front geometries are observed [10]. Compared to a non-halide process, a halide process principally offers an advantage of a high lateral-to-vertical growth rate ratio [12], which is a key factor influencing the growth front and related dislocation motion in the GaN epilayer grown by ELO. HVPE offers a high growth rate and high material quality for $\mathrm{GaN}$ growth $[13,14]$. The typical growth rate can get $100-200 \mu \mathrm{m} / \mathrm{hr}$. GaN produced by the HVPE technique does result in a greatly reduced intensity of the defect-based luminescence referred to as the yellow band (YL) when compared to the trimethyl gallium (TMG)-based MOVPE materials. The ability to grow thick GaN film with high quality and obtaining low TD density in ELO GaN films make it possible to get high quality self-standing GaN wafer in a single HVPE 
growth chamber. In this paper the structural properties of HVPE ELO GaN films, especially coalesced flat-top ELO GaN films, are investigated systematically. The tilting of TDs from the vertical orientation to horizontal or inclined orientations in the GaN "seeding layer" is observed. The lattice tilt due to pileup of tilted TDs is found and the tilt angle is determined. This tilt angle is dependent on ELO growth conditions.

\section{EXPERIMENTS AND RESULTS}

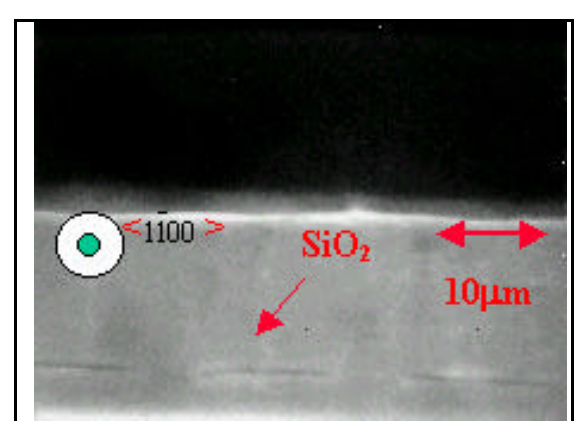

Fig. 1 Cross-sectional SEM picture of a coalesced flat-top ELO GaN sample grown by HVPE. There is no void observed at the coalescence interface.
The GaN 'substrate' for ELO growth is an MOVPE grown GaN film on a (0001) sapphire substrate coated with a $\sim 100 \mathrm{~nm}$ thick patterned $\mathrm{SiO}_{2}$ layer. The pattern includes an array of $2-4 \mu \mathrm{m}$ parallel stripe windows openings on a 12 $\mu \mathrm{m}$ period oriented along the $\langle 1 \overline{1} 00\rangle$ orientation. The subsequent ELO growth of $\mathrm{GaN}$ is performed in a vertical HVPE system. In the HVPE process, the growth temperature is varied over the range of $1030^{\circ} \mathrm{C}$ to $1100^{\circ} \mathrm{C}$. The input V/III ratio is controlled between 33 to 83 and the $\mathrm{NH}_{3}$ mole fraction is over the range of $\left[\mathrm{NH}_{3}\right]=0.076-0.12$. The nitrogen is used as the carrier gas. The operational pressure during the ELO growth is the atmospheric pressure.

The structural properties of the HVPE ELO GaN films are studied by using transmission electron microscope (TEM), scanning electron microscope (SEM) and high resolution X-ray diffraction (XRD). Atomic force microscope (AFM) is employed to characterize the surface morphology of the ELO GaN films. Fig. 1 shows a crosssectional SEM picture of a coalesced flat-top HVPE ELO $\mathrm{GaN}$ sample. The growth temperature is $1100^{\circ} \mathrm{C}$ and $\left[\mathrm{NH}_{3}\right]=0.076,[\mathrm{HCl}]=0.0023$. It could be seen from the figure that the top is flat and the coalescence is perfect. There are no observable voids found at the coalescence interface.

Fig. 2 is an AFM image of the surface morphology in the ELO GaN area of the sample shown in Fig. 1, which clearly shows features characteristics of step-flow growth. There is no detectable step termination in the measured ELO GaN area, indicative of a low TD density

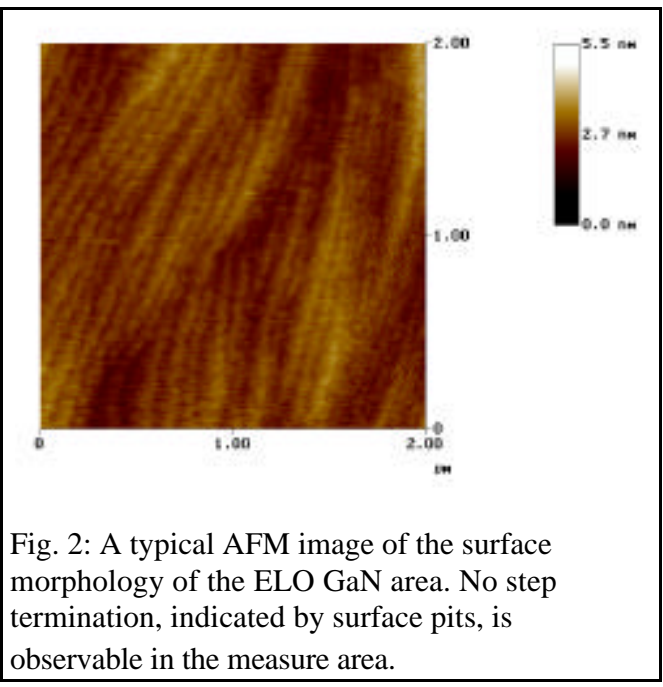




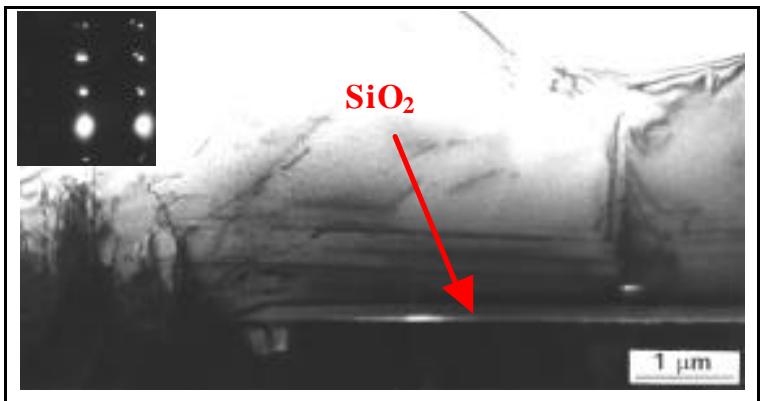

Fig. 3 A cross-sectional TEM picture of the HVPE ELO $\mathrm{GaN}$ film. The change in the direction of the TDs is observable in the picture. The insert is an electron diffraction pattern in the same area. Two sets of diffraction spots indicate that there is a lattice tilt in the ELO area due to pileup of TDs.
[15].

Fig. 3 presents a cross-sectional TEM picture of the same sample. The insert is an electron diffraction pattern in the same area. From Fig. 3, most TDs propagated from the MOVPE-grown GaN "seeding layer" along caxis and bend from the vertical during ELO growth. This change in dislocation motion results in a reduced TD density zone over the masking regions of the ELO GaN film. A lattice tilt results

from this change in dislocation direction leading to a new series of diffraction spots in the electron diffraction pattern. The angle between two spot lines is the angle of lattice tilt. In order to precisely determine this tilt angle, high- resolution $\omega$-scan XRD measurements were performed as shown in Fig. 4. In Fig. 4, two separate, approximately symmetric, diffraction peaks appear on two sides of the major diffraction peak. The angle separation of the "tilt" diffraction peak to the major diffraction peak is $\sim 3.3^{\circ}$. In this case, the fullwidth-at-half-maximum (FWHM) of the major diffraction peak is 643 arcsec. The

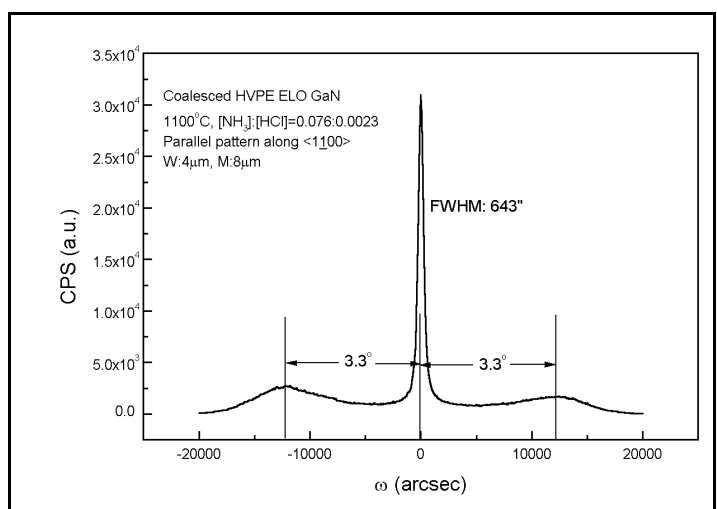

Fig. 4: High-resolution XRD rocking curve of the HVPE ELO GaN sample shown in Fig. 1. Two separated "tilt" diffraction peaks appear on both side of the major diffraction peak. asymmetry of two "tilt" diffraction peaks may result from a slight misalignment in the XRD measurement.

Modifications to the ELO growth conditions can alter the TD distribution within the ELO films, as indicated by the intensity of the "tilt" diffraction peaks and their angle spacing from the major diffraction peak. Fig. 5 is a $\omega$-scan XRD rocking curve of a HVPE ELO GaN sample grown using a $\left[\mathrm{NH}_{3}\right]=0.12$, $[\mathrm{HCl}]=0.0022$. From Fig. 5, two "tilt" diffraction peaks could be seen again as two wings of the major diffraction

peak at an angle of $\sim 2.4^{\circ}$, as compared to $\sim 3.3^{\circ}$ in the case of lower V/III ratio shown in Fig. 4. The FWHM of the major diffraction peak also decreased to 479 arcsec. The improved structural properties of the crystal coincide, in general, with conditions that 


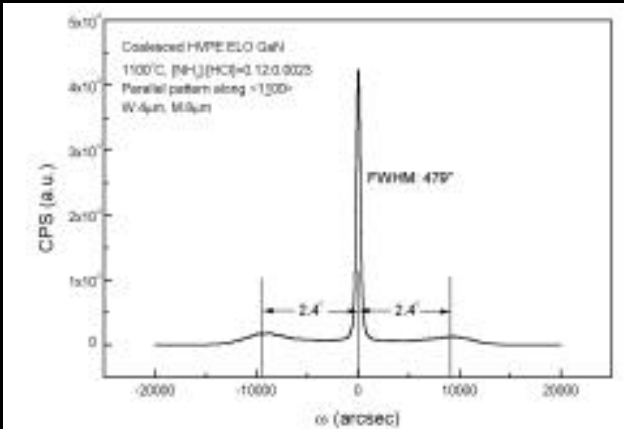

Fig. 5: High-resolution XRD rocking curve of the HVPE ELO GaN sample grown with mole fractions of $\left[\mathrm{NH}_{3}\right]=0.12,[\mathrm{HCl}]=0.0022$. Again, two separated "tilt" diffraction peaks appear on both side of the major diffraction peak. lead to improved structural properties in non-patterned growth. This idea has been proved by TEM analysis. The TEM image of the HVPE ELO GaN sample shown in Fig. 5. reveals that many of the TDs initiating in the window area bend into the plane of growth or thread through the ELO region at an oblique angle. The ELO region, particularly in the regions far from the opening windows appear to have a low TD density and should give rise to a reduced XRD linewidth. No observable void is found at the coalescence interface in this case, too.

\section{DISCUSSION}

The resulting structure during the ELO process is known to be dependent on the gas phase transport of reactants to the growth front, which is dependent on the growth conditions and mask geometry. The growth chemistry also plays an important role in determining the local growth rates and local V/III ratio. These factors have been shown to have a primary influence on the facet formation in MOVPE growth. The addition of chloride-based species into the growth ambient will decrease the driving force or supersaturation and thus decrease the overall growth rate [12]. Reduced supersaturation has often been associated with higher surface transport lengths on the growth front. The specific supersaturation at the growing facet will have this additional factor, i.e. local supersaturation, which affects the local facet determination. The enhanced lateral growth rate of HVPE ELO technique assists in the elimination of the coalescence void at the merge interface [12].

The mechanism of the TD bending in the HVPE ELO region is unknown. The influence of a free-bounding surface on the motion of dislocations can play a role. In addition local strains arising from the initial strain state of the $\mathrm{GaN}$ "seeding layer" and the thermal expansion difference-based strains due to the presence of the masking materials will affect dislocation motion by developing local strain gradients in the film. The high lateral-to-vertical ratio and high rate of growth in HVPE, when compared to MBE or MOVPE, aid in the development of a merged front free of a coalescence void. Increased V/III ratio lead to improved materials properties, while maintaining a void-free coalescence, in ELO as in the non-masked growth.

\section{CONCLUSIONS}

The dependence of the structural properties of HVPE ELO GaN films on gas phase V/III ratio has been investigated in this paper. A lower V/III ratio was found to lead to improved ELO materials, as determined by XRD measurement. No observable voids were present at the coalescence interface. A lattice tilt is seen in all of the HVPE samples 
characterizing the deflection of the <0001> planes between the GaN grown over the masking region and the initial window opening.

\section{ACKNOWLEDGEMENT}

R. Zhang, Y, Shi, Y.G. Zhou, B. Shen, Y.D. Zheng thank the financial support of the China "863" national high-tech development program and National Natural Science

Foundation of China with contracts \#69976014, \#69636010, \#69806006, \#69987001. The authors would like to acknowledge the financial support of the ONR MURI on Compliant Substrates, and the facilities support of the NSF Materials Research Science and Engineering Center on Nanostructured Materials and Interfaces, USA.

\section{REFERENCES}

1. S.Nakamura, M.senoh, S.Nagahara, N.Iwasa, T.Yamada, T.Matsuahita, H.Kiyoku and Y.Sugimoto, Jpn.J.Appl.Phys. 135, L74 (1996).

2. S.Nakamura, M.senoh, S.Nagahara, N.Iwasa, T.Yamada, T.Matsuahita, H.Kiyoku, Y.Sugimoto, T.Kozaki, H.Umemeoto, M.Sano, and K.Chocho, Appl.Phys.Lett, 72, 2014 (1998)

3. S. J. Pearton, J. C. Zolper, R. J. Shul, F. Ren, J. Appl. Phys., 86, 1(1999).

4. F.A. Ponce and D.P. Bour, Nature, 386, 351(1997).

5. H. Marchand, X.H. Wu, J.P. Ibbetson, P.T. Fini, P. Kozodoy, S. Keller, J.S. Speck, S.P. DenBaars, and U.K. Mishra, Appl. Phys. Lett. 73, 747 (1998).

6. Michael E. Coltrin, Christine C. Willan, Michael E. Bartram, Jung Han, Nancy Missert, Mary H. Crawford, Albert G. Baca, MRS Internet J. Nitride Semicond. Res. 4S1, G6.9(1999).

7. A. Sakai, H. Sunakawa and A. Usui, Appl. Phys. Lett., 71, 2259(1997).

8. O-H. Nam, M.D. Bremser, T.S. Zheleva and R.F. Davis, Appl. Phys. Lett., 71, 2638(1997).

9. H. Matsushima, M. Yamaguchi, K. Hiramatsu and Sawaki, Proc. 2 nd Int. Conf. Nitride Semiconductors, Tokushima, Japan, 1997, p492.

10. R. Zhang, L. Zhang, D.M. Hansen, Marek P. Boleslawski, K.L. Chen, D.Q. Lu, B. Shen, Y.D. Zheng and T.F. Kuech, MRS Internet J. Nitride Semicond. Res. 4S1, G4.7 (1999)

11. Yasutoshi Kawaguchi, Shingo Nambu, Hiroki Sone, Masahito Yamaguchi, Hideto Miyake, Kazumasa Hiramatsu, Nobuhiko Sawaki, Yasushi Iyechika and Takayoshi Maeda, MRS Internet J. Nitride Semicond. Res. 4S1, G4.1 (1999).

12. J-O Carlsson, Solid State \& Mater. Sci., 16, 161(1990).

13. N.R. Perkins, M.N. Horton, Z.Z. Bandic, T.C. McGill and T.F. Kuech, Mat. Res. Sci. Symp. Proc. 395, 243 (1996).

14. R.J. Molnar, K.B. Nichols, P. Maki, E.R. Brown and I. Melngailis, Mater. Res. Soc. Symp. Proc., 378, 479 (1995).

15. H. Marchand, J.P. Ibbetson, Paul T. Fini , Peter Kozodoy, S. Keller, Steven DenBaars, J. S. Speck, U. K. Mishra, MRS Internet J. Nitride Semicond. Res. 3, 3(1998). 